\title{
An Application Framework to Support Information Sharing in Collaborative Work
}

\author{
Markus Kolland, Michael Berger \\ Siemens AG, Corporate Technology Department, \\ Otto-Hahn-Ring 6, 80179 Munich, Germany, \\ Phone +4989636 41343, Fax +498963640757, \\ eMail \{Markus.Kolland,Michael.Berger\}@zfe.siemens.de
}

\begin{abstract}
This paper describes COCOON, an object-oriented application framework which facilitates the construction of software systems for computer supported collaborative work (CSCW). Within a simple and orthogonal programming model COCOON provides a variety of mechanisms which support the implementation of functionality for access, manipulation and consistency of shared information in the context of typical collaboration patterns (synchronous, asynchronous and autonomous interactions). The focus of this paper are aspects which are of specific interest for the support of autonomous collaboration.
\end{abstract}

\section{Keywords}

CSCW, Telecooperation, Application Frameworks for CSCW, Information Sharing, Consistency of Shared Information, Autonomous Collaboration

\section{INTRODUCTION}

CSCW is an application domain which represents one of the driving forces behind the information superhighway and becomes increasingly important for companies and organizations doing business on a world-wide basis. The implementation of CSCW systems however is extremely complex. The major source of this complexity lies in the provision of functionality for access, manipulation and consistency of shared information in the context of typical CSCW environments which are characterized by a variety of information sharing patterns, by concurrent activities and geographically dispersed, mobile hardware components (Blair 1991).

COCOON is an application framework to facilitate the construction of CSCW applications. It provides a simple information-, communication and consistency model which supports typical human-human collaboration patterns. In this paper we focus on a collaboration pattern called autonomous work, in which group information can be altered while group members are disconnected from each other (Kistler 1993). Given such a scenario the different views on the 
shared group information can diverge and have to be integrated into one consistent view when the autonomous member is reconnected. COCOON supports such autonomous collaboration providing an automatic reintegration, conflict detection and conflict resolution strategy which minimizes information loss after reintegration while guaranteeing a CSCW specific notion of consistency.

\section{COCOON ARCHITECTURAL OVERVIEW}

In COCOON a local instance of the CSCW application resides on every node on which a group member participates in the respective collaborative task (e.g. code inspection). These application instances interact in a joint context, called a session. Each session provides a shared information space in which application instances can create, delete or manipulate objects. Each application instance has access to a local replica of the shared information space (Greenberg 1991).

Communication is done implicitly by applying update operations to the shared information space. Update operations are forwarded to each application instance in the session. They are applied to the local copy of the shared information space.

The status of the shared information space replicas can diverge when concurrent interactions occur, messages are delayed/get lost or when session members work autonomously. In CSCW applications for which this behavior causes consistency problems the mechanisms of the COCOON consistency model can be deployed. They ensure that the differences between the shared information space replicas stay within semantically well-defined boundaries. COCOON allows to choose between various such boundaries reflecting different notions of consistency in human-human collaboration.

\subsection{The COCOON Information Model}

The COCOON information model is hypertext-like (Reinhard 1994). An object represents an information entity with attributes holding its content and links representing relationships. In order to allow uniform access to all objects in a distributed environment each object has a session wide, unique identifier (OID). OIDs are specified by the application when objects are created in the shared information space. COCOON provides five operations to manipulate objects :

- The createobj (InfoSpace, OID, Obj) operation inserts the local object 'Obj' under the unique identifier 'OID'

- The deleteobj(InfoSpace, OID) operation deletes the object with unique identifier 'OID'.

- The createlink (InfoSpace, OID1, OID2, Linkname) operation establishes a (unidirectional) link between two objects. The name under which this link is established is denoted by 'Linkname'.

- The deletelink (InfoSpace, OID, Linkname) operation deletes a link.

- The changeAttr (InfoSpace, OID, AttrName, Obj) operation changes an attribute value of an object.

- The addAttr(InfoSpace, OID, AttrName, Obj) operation adds the value 'Obj' to a set-oriented attribute. 
- The delAttr (InfoSpace, OID, AttrName, Obj) deletes the value 'Obj' from a set-oriented attribute.

In analogy with the definition in the object-oriented database field, COCOON information spaces are equivalent if they contain the same objects and these objects have identical attributes and associations (Dittrich 1991).

\subsection{The COCOON Communication Model}

\section{Synchronous and Asynchronous Communication}

Communication is implicit via updating the shared information space and broadcasting these updates to all application instances in a session. Synchronization between the sending and the receiving application instances depends on the interaction model which is implemented by the CSCW application. COCOON supports synchronous, asynchronous and autonomous interaction patterns (Ellis 1991).

A history is defined as a sequence of update operations. Histories record in chronological order the sequence of update operations applied to a session members local copy of the shared information space. Two histories are equivalent if applying them to a shared information space results in two equivalent shared information spaces. For a formal definition we refer to (Kolland 1995).

\section{Autonomous Communication}

The autonomous communication model supports situations within collaborative work in which session members are temporarily disconnected from each other, either deliberately ('don't disturb') or as a result of a communication breakdown. Figure 1 depicts the histories of three application instances in a session after a phase of synchronous and autonomous collaboration :

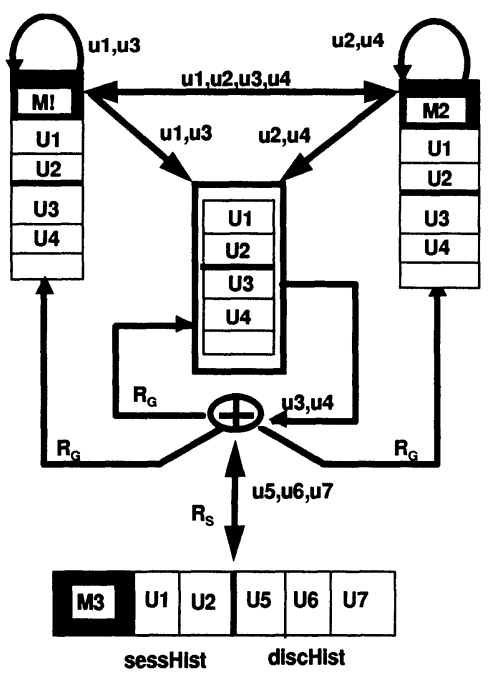

Figure $1:$ The autonomous communication and consistency model. 
At the beginning of the session (synchronous interaction) updates $u_{1}$ and $u_{2}$ are initiated by application instance $m_{1}$ and $m_{2}$ respectively. Each update is broadcasted to the other application instances. After $u_{2}$ has been received by every member, $m_{3}$ is disconnected and enters the autonomous state. In this state $m_{3}$ initiates updates $u_{5}$ and $u_{6} . m_{1}$ initiates $u_{3}, m_{2}$ initiates $u_{4}$. Operations $u_{3}, u_{4}, u_{5}$ and $u_{6}$ which can not be forwarded to all other application instances are transparently logged in the respective histories. When reconnection is initiated by $m_{3}$, the histories accumulated during the autonomous phase at the singleton and the group members have to be merged in order to restart synchronous collaboration with consistent shared information space replicas (Davidson 1985).

\section{A CONSISTENCY MODEL FOR AUTONOMOUS COLLABORATION}

\subsection{A Consistency Notion for Autonomous Collaboration}

But what does consistency of shared information space replicas in this context mean ? Two basic requirements of collaborative work must be fulfilled by the consistency notion for autonomous work (Kolland 1995) :

- EQUIV - The histories of group and singleton from the point where the disconnection started up to the point where reconnection is finished must be equivalent.

- MERGE - Updates made by both the group members and by the singleton member during the autonomous phase must be reflected in the resulting histories as long as this does not conflict with the EQUIV condition.

\subsection{The Reconnection Algorithm}

The COCOON reconnection algorithm is based on the construction of replay lists $R_{S}$ and $R_{G}$. $R_{S}$ is applied to the singleton and $R_{G}$ to the group members at reconnection time. The construction algorithm fulfills the conditions EQUIV and MERGE. This is achieved by incorporating both the group operations in $R_{S}$ and the singleton operations in $R_{G}$ while detecting and resolving conflicting operations. The following algorithm illustrates the overall strategy for constructing the replay lists $R_{S}$ and $R_{G}$ :

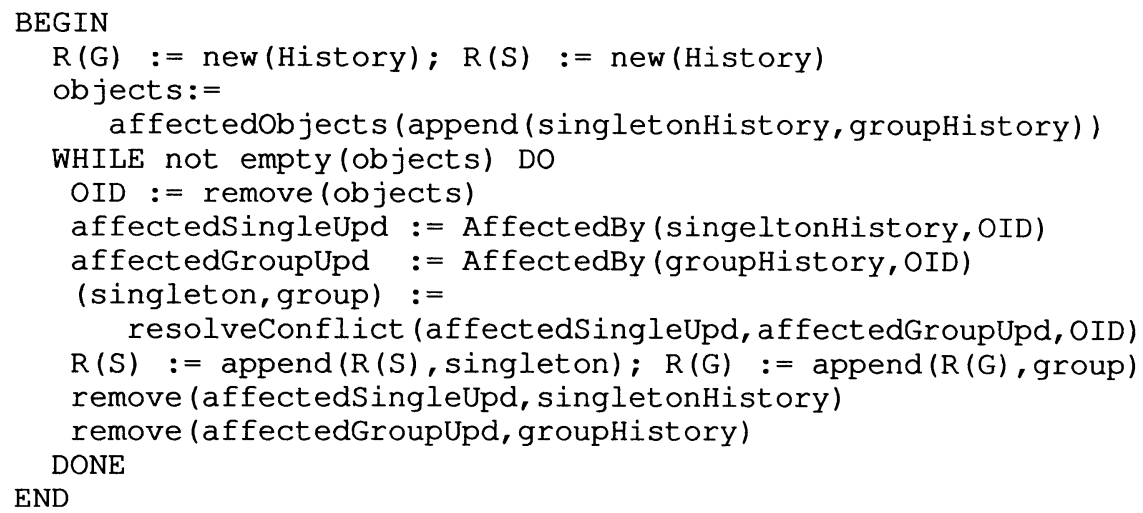


At the beginning $R_{S}$ and $R_{G}$ are empty. From both histories the objects which have been manipulated in the course of the autonomous phase are extracted. For each of these objects a pair of histories which contain operations on this object in the singleton and in the group history respectively is extracted. To each of these pairs, a three step conflict resolution algorithm is applied an the resulting replay operations are appended to $R_{S}$ and $R_{G}$ respectively.

\subsection{Conflict Resolution}

COCOON considers all write-operations including creation/deletion of objects as potentially conflicting since each delete/create/update on one side may conflict with any operation on the other side semantically. The semantics of an operation is however up to the application and therefore such conflicts can not be detected by the COCOON reconnection algorithm. They have to be resolved by the application itself. This however is only possible, if the effect of such operations can be reversed after reconnection. COCOON operations fulfill this requirement.

In case of a change to the same attribute of an object on both sides, the resolution algorithm has to decide between the update of one side either according to a 'group-has-it-right' or a 'singleton-has-it-right' policy. The respective policy can be selected by the application at reconnection time.

\section{Conflict Resolution Step 1}

The first step classifies the two conflicting histories, compares the signatures and reduces them to operations which only manipulate attributes or associations. The following signatures are identified (regular expression notation is used - \{ Rest \} denotes the whole set of COCOON operations without operations createObj and deleteObj):

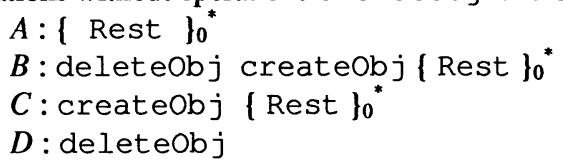

EQUIV and MERGE are maintained by inserting operations into $R_{S}$ and $R_{G}$ according to the following scheme :

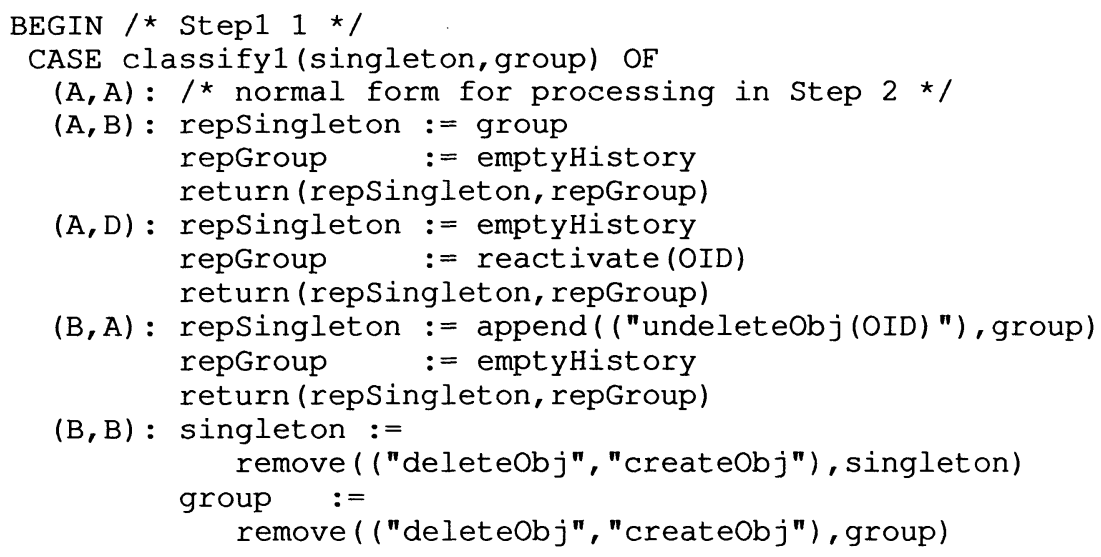




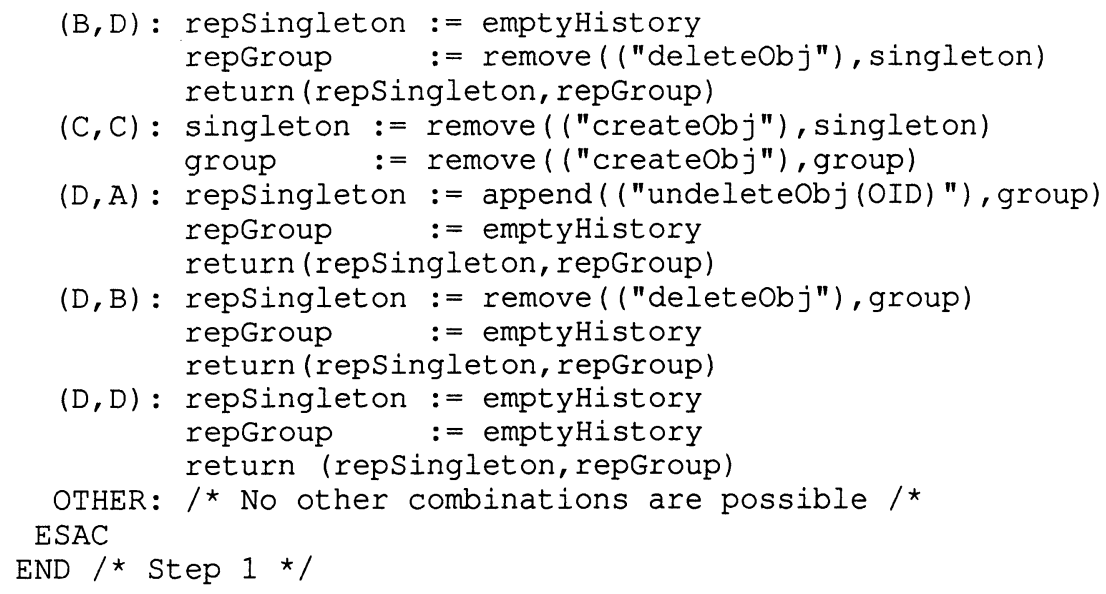

AxB : The group deletes the existing object and creates a new object with the same 'OID' while the singleton works on the original copy. Only one state of the object however is possible after reconnection. Assuming a 'group-has-it-right' policy the object has to be deleted at the singleton and the complete group history on this object has to be replayed. The singleton replay history is therefore identical with the group history for the respective object. The singleton operations are discarded and the conflict resolution algorithm terminates for the respective object.

AxD : In this case the group deletes the object while the singleton continues working on it. Given the condition MERGE the operations on the object made by the singleton must be preserved. Therefore reactivate () assembles the history to be replayed at the group from the state of the object at the singleton. The delete operation in the group history is discarded and the conflict resolution algorithm terminates for the respective object.

BxA : This case is symmetric to AxB. The singleton deletes the object and creates an object with the same 'OID' while the group works on the original copy. In order to be able to solve such a conflict a shadow copy is created before a singleton deletes an object. The operation undelete (OID) restores the object to the state before deletion.an the group updates have to be replayed. All singleton operations are discarded and the conflict resolution algorithm terminates for the respective object.

BxB : In this case singleton and group delete and create an object with the same 'OID' independently from each other and update the object. By discarding operations deleteobj and createObj histories of the form AxA are created. Histories of this (normal) form are processed in step 2 of the conflict resolution algorithm. For this transformation step no replay operations are necessary.

BxD: In this case singleton and group delete the object, the singleton however reinstantiates and updates an object with the same 'OID'. According to the condition MERGE all operations carried out by the singleton (despite deleteObj) have to be replayed at the group. The 
groups deleteObj operation is discarded and the conflict resolution algorithm terminates for the respective object.

CxC : This case is analogous to $\mathrm{BxB}$. Both sides create an object with the same 'OID' independently from each other and update it. Both createObj operations are discarded resulting in signatures of the form AxA. These histories are processed in step 2 of the algorithm.

DxA : This case is symmetric to AxD. The singleton deletes the object while the group continues working on it. To solve this conflict the shadow copy, which has been created by the singleton before deleting the object has to be restored. This is done by replaying the special operation undelete (OID). Then the operations initiated by the group are replayed. The deleteObj operation of the singleton is discarded and the conflict resolution algorithm terminates for the respective object.

DxB : This case is symmetric to $\mathrm{BxD}$. At the singleton the complete group history, despite the deleteObj operation has to be replayed and the conflict resolution algorithm terminates for the respective object.

DxD : In this case no specific conflict resolution is necessary. No replay operations are constructed and the conflict resolution algorithm terminates for the respective object.

\section{Conflict Resolution Step 2}

The reduced histories from step 1 are classified, compared and the replay operations to resolve conflicts are generated. The following signatures are relevant :
A : $\{\text { addAtt } r \mid \text { delAtt } r\}_{0}{ }^{*}$
B : changeAttr

Replay operations are constructed according to the following scheme :

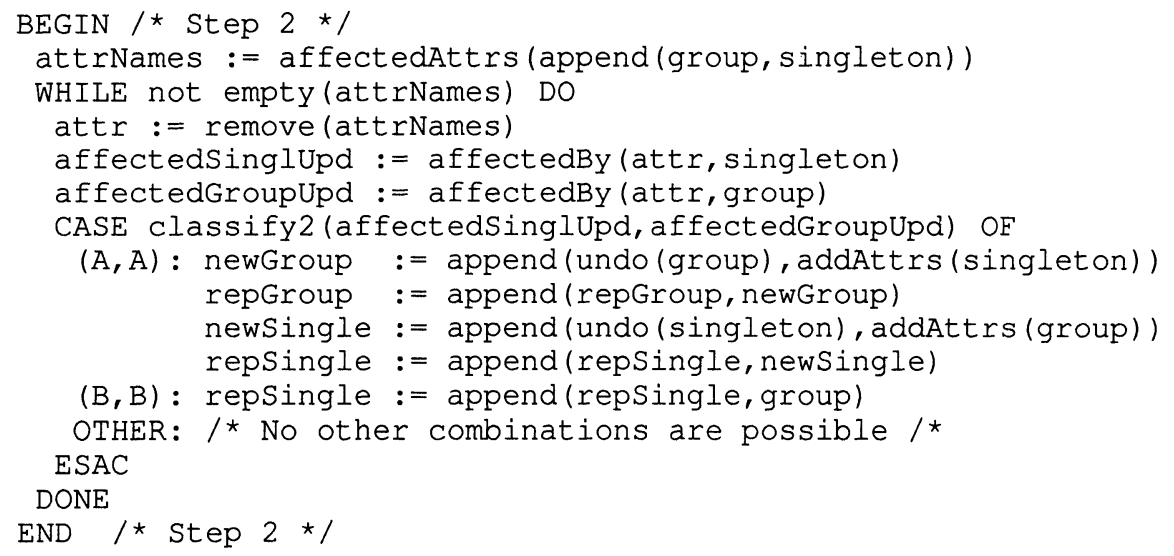


AxA : On both sides only operations with 'add' semantics have been applied to the respective attribute. According to the condition MERGE however all updates have to be preserved despite this is in conflict with the condition EQUIV. To achieve MERGE semantics the deleteAttr operations in both histories have to be undone. This is achieved by the function undo () which constructs the respective undo-operations for each history. The sequence of addAttr operations of the opposite side is then appended to the replay history of singleton and group respectively. Given the set semantics of attributes this yields a correct result with respect to EQUIV and MERGE.

BxB : On both sides only operations with 'overwrite' semantics have been applied to the respective attribute. To fulfill the condition EQUIV one of the operations has to be selected for both sides. Assuming a 'group-has-it-right' policy the respective group operation has to be appended to the singleton replay history. The singleton operation is discarded.

\section{Conflict Resolution Step 3}

In the third step conflicts on associations are resolved with a simplified version of the algorithm in step 2, since only operations of type AxA are allowed on associations.

The replay lists constructed in steps 1-3 are returned as result of the resolveConflict () function in the algorithm of section 3.2.

\section{IMPLEMENTATION}

COCOON is deploying a replicated architecture in order to support interactive applications over slow WAN connections and autonomous work on mobile components. The content of a shared information space is replicated at each applications instances site. To implement the described communication and consistency models we needed a communication platform with a group-notion and a broadcast facility including strong message ordering guarantees. for the implementation of the reconnection algorithm, the virtual synchrony model (Garcia-Molina 1991) in which messages and group membership changes are ordered consistently was a prerequisite.

The ISIS/News platform (Birman 1993) fulfilled all criteria. An orthogonal integration with the Smalltalk programming environment was achieved by extending the Smalltalk dependency mechanism to remote objects and object groups using ISIS/News as message transport.

\section{CONCLUSION}

In order to validate the COCOON approach, we implemented CoNus, a multi-purpose CSCW toolkit (Berger 1996). The shared information space abstraction, the communication models and especially the support for autonomous work and consistent reintegration facilitated the implementation of the application considerably.

Generally speaking we were able to prove that with the COCOON application framework it is possible to hide many of the complexities of CSCW application design. Thus CSCW applications can be prototyped and implemented much more rapidly containing more advanced collaboration functionality as in traditional design approaches. 


\section{REFERENCES}

Berger, M. (1996) CoNus - A CSCW system supporting synchronous, asynchronous and autonomous collaborative work, Proceedings of the International Conference on Distributed Platforms, Dresden

Birman, K. (1993) The Process Group Approach to Reliable Distributed Computing, Communications of the ACM, 36(12)

Blair, G. Rodden, T. (1991) The Impact of CSCW on ODP, Proceedings of the Workshop on Open Distributed Computing (ODP), Berlin

Davidson, S. Garcia-Molina, H. Skeen, D. (1985) Consistency in Partitioned Networks, ACM Computing Surveys, 17(3)

Dittrich, K. (1991) Object-Oriented Database Systems, Topics in Information Systems, Springer-Verlag

Ellis, C.A. Gibbs, S.J. Rein, G. (1991) Groupware - Some Issues and Experiences, Communications of the ACM, 34(1)

Garcia-Molina, H. Spouster, A. (1991) Ordered and Reliable Multicast Communication, ACM Transactions on Computer Systems, 9(3)

Greenberg, S. (1991) Liveware - A new Approach to Sharing Data in Social Networks, International Journal of Man-Machine Studies, 34(3)

Kistler, J.J (1993). Disconnected Operation in a Distributed File System, Ph.D. Dissertation, Carnegie Mellon University

Kolland, M. (1995) Generische Unterstützung von Infomrmation Sharing im Kontext computer-unterstützter Gruppenarbeit, Ph.D. Dissertation, Technical University Munich (in German)

Reinhard, W. Schweitzer, J. Voelksen, G. (1994) CSCW Tools : Concepts and Architecture, IEEE Computer, 27(5) 